\title{
Position Detection of Moving Objects Using a Two Dimensional PSD and the Ceiling LED Lighting
}

\author{
Kenta Kagemoto, Kosuke Onishi, Shiyuan Yang, \\ Kyushu Institute of Technology, 1-1 Sensui-cho Tobata-ku Kitakyushu-shi Fukuoka-ken, 804-8550, Japan \\ *Corresponding Author: q349406k@mail.kyutech.jp
}

\begin{abstract}
In recent years, the places where a robot plays an active part in various scenes increase, we think that the places which need robot increase in the future. Position detection of the moving objects such as robots itself is very important. The method of the conventional position detection uses Global Positioning System (GPS) and a camera. Each method has some disadvantages. For example, large error, long processing time, and usable place.

Therefore, we propose a new method of position detection of the moving objects using a two-dimensional Position Sensitive Device (2D PSD) and the ceiling Light Emitting Diode (LED) lighting in this study. In this method, we establish the LED whose frequency is modulated in a room, and defines positional coordinates of each LED in advance. 2D PSD can receive plural light at the same time by getting the spectrums every frequency using FFT. The plural light is incident on PSD during movement in various places. We choose four LED which are the nearest to PSD from that and perform position detection using them. Accordingly, we can relatively detect the position of the 2D PSD by detecting the position of the LED with a 2D PSD. This method is accurate and available under various situation.

We performed the experiments of position detection and showed the effectiveness of this method.
\end{abstract}

Keywords: 2D PSD, LED, Frequency, Position detection

\section{Introduction}

In recent years, the places where a robot plays an active part in various scenes increase, we think that the places which need robot increase in the future. For example, a robot that will automatically carry various things in the room such as a hospital, a robot that moves put a handicapped human foot, a robot who will help of everyday life in those very useful if spread is there. In an indoor automatic mobile robot, the position detection of robot oneself is very important in that. If a robot do not know the position of its own correctly, it cannot correspond to the various situations which can happen, and it isn't possible to arrive to the destination. Therefore, it is necessary to always grasp where a robot is to move exactly at all places. There are some conventional methods of position detection, but there are some disadvantages in that methods.

We show some example. The first is a position detection using the Global Positioning System (GPS) and radio waves from a base station. This method can detect the rough position, but an error becomes big by using the signal from the far-off place. It is not possible to use this method at the place where a radio wave cannot be used. Next, the method to acquire a picture from cameras and detect the position of the robot by image processing ${ }^{(1)(2)}$. Position detection by image processing is the method of the detecting a position by pattern matching using the images taken in real time and the image was taken in advance, and the method of the detecting a position from the appearance of landmarks that are installed in advance. The former is time-consuming because it is necessary to prepare various images in advance. In addition, it is impossible to correspond to inclination. In the latter, if a robot is wherever, the appearance of the landmark must be different. Also it takes time because complicated calculation is needed by position detection using image processing. The method of this conventional position detection has a demerit.

Therefore, we propose a new method of position detection using a two-dimensional Position Sensitive Device (2D PSD) and the ceiling Light Emitting Diode (LED) lighting in this study. In this method, we modulate plural LED to different frequency. We establish the LED whose frequency 
is modulated in a room, and defines positional coordinates of each LED in advance. Accordingly, we can relatively detect the position of the 2D PSD by detecting the position of the LED with a 2D PSD. We can overcome the disadvantages of the method conventionally by using this method.

\section{Principle}

\subsection{D PSD}

We described 2D PSD. 2D PSD is a light sensor which can detect the position of the spot-like light. Basically it has a PIN structure with a single joint surface as a photodiode. An electric charge occurs when we give a spot of the light on a semiconductor surface, and the electric charge that occurred arrives at the electrode of both ends. The quantity of the electric charge that arrived at it is inversely proportional to the distance from the position of the spot light to an electrode. We can obtain position data of the spot light by performing a necessary calculation using the electric current which we took out of an electrode.

In this study, the output electric current from 2D PSD is signal after it was converted into the voltage. Because, we use the 2D PSD module which a current - voltage conversion circuit is incorporated in. Four output voltage $V_{X 1}, V_{X 2}, V_{Y 1}$, and $V_{Y 2}$ represents the position information, if the width of the light-receiving surface is $\mathrm{L}$, the position coordinates $x, y$ can be calculated by the following equation.

$$
\begin{aligned}
& x=\frac{\left(V_{X 2}+V_{Y 1}\right)-\left(V_{X 1}+V_{Y 2}\right)}{\left(V_{X 1}+V_{X 2}+V_{Y 1}+V_{Y 2}\right)} \times \frac{L}{2} \\
& y=\frac{\left(V_{X 2}+V_{Y 2}\right)-\left(V_{X 1}+V_{Y 1}\right)}{\left(V_{X 1}+V_{X 2}+V_{Y 1}+V_{Y 2}\right)} \times \frac{L}{2}
\end{aligned}
$$

It is possible to reliable position recognition and very fast response, because 2D PSD sensor is simple operation. In addition, it is a sensor having high precision because resolving power is very high.

\subsection{Position detection of moving objects}

We show the flow of the system in the position detection of moving objects in Fig.1. In this study, we modulate plural LED to different frequency. We establish the LED whose frequency was modulated in a room, and defines positional coordinates of each LED in advance. These are usually used as indoor lighting. We can detect a position of plural LED at the same time by using LED of the different frequency ${ }^{(3)}$. Distinction of ambient light and the light of the LED is possible by performing frequency modulation. The light from the LED is condensed by using an optical lens, and focus on the light-receiving surface. We show this in Fig. 2.

In Fig. 2, ' $d$ ', is the distance from 2D PSD to the lens, " $h$ " is the distance from the LED to the lens. Thus 2D PSD receives the light, and detects the coordinates of each LED by using the equation (1) (2). After having received light, we perform Analog/Digital (A/D) conversion of the output from 2D PSD with AD converter. Then the spectrum of the output waveform in the modulation frequency is obtained by performing a Fast Fourier Transform (FFT) on the Personal Computer (PC). And it detect the coordinates make the necessary calculation.
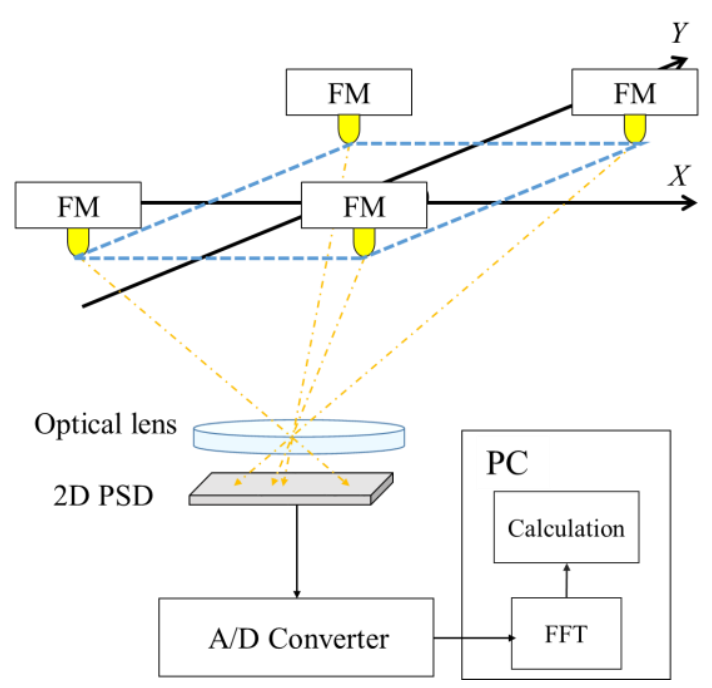

Fig.1. System configuration

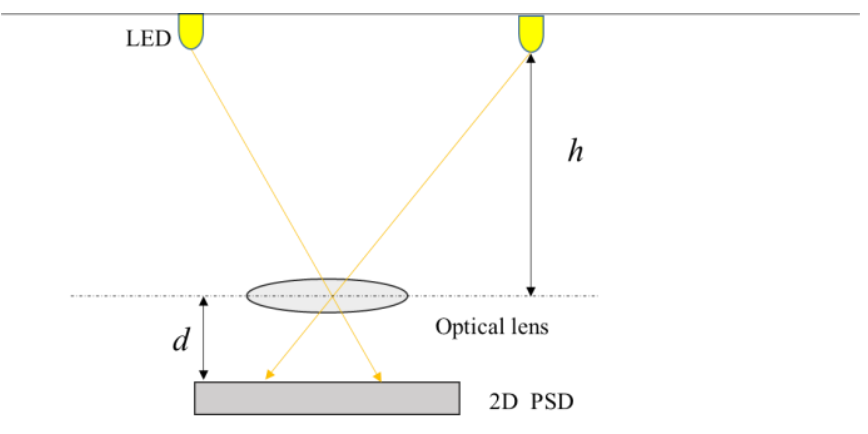

Fig. 2. State of the light receiving 


\subsubsection{Rough position detection}

Even if there is the moving objects which carried PSD in any place, it allows light of four LED to be subjected to light at the same time. When a PSD receive light more than four, the coordinate which measured using the coordinate detection to show in 2.2.2 uses four LED that PSD is near. We know it which LED there is the moving objects under by a combination of frequency of four received LEDs. It can recycle the same frequency by changing a combination of four frequency. Therefore, it can be used in a wide range.

\subsubsection{Accurate position detection}

Value $x, y$ obtained from PSD is a coordinate on the light receiving side. Detects the LED coordinates relative to the 2D PSD using this value. It uses triangular resemblance relations. We show a figure of principle in Fig. 3.

Value $X a, Y a$ is the coordinates relative to 2D PSD. $d$ is the distance from the LED to the lens. The two triangles are similar. The same applies to the $y$-coordinate. Therefore, it is possible to obtain an LED coordinates $\mathrm{Xa}, \mathrm{Ya}$ relative to 2D PSD by the following equation.

$$
\begin{aligned}
& X a=\frac{h}{d} x \\
& Y a=\frac{h}{d} y
\end{aligned}
$$

Among the light received, performs these measurements using four LED close to the PSD. Because it use four LED, we can perform position detection even if there is an abnormality in one LED.

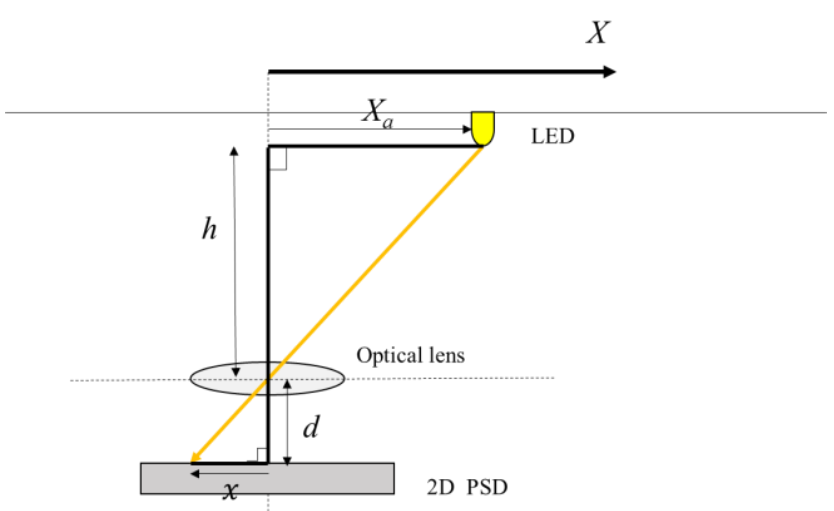

Fig.3 Principle of detect the LED coordinate

\section{System configuration}

The system consists of the light projecting side and the light receiving side. The light projecting side is the LED whose frequency was modulated. It consists of a frequency modulation circuit using a function generator IC and a wide-angle high brightness LED. The light receiving side consists of an optical lens and 2D PSD. We show this series of flow in Fig. 4.

\subsection{Frequency modulation circuit and LED}

We use LED directivity angle is large, it is $140\left[^{\circ}\right]$. We use a function generator IC for a frequency modulation circuit, it can change the amplitude, frequency, the voltage by regulating the semifixed resistance in the circuit.

\subsection{Configuration of the light receiving side}

The light receiving side consists of an optical lens, 2D PSD, A/D converter and PC. The projected light is condensed by optical lens, and imaged on the light receiving side of 2D PSD. The electric current which accepted the position on the light receiving side of received light is converted into the voltage, and it is output by two-dimensional PSD. After that, A/D converts the output from 2D PSD by an A/D converter and sends those data to the PC. We perform FFT of the digital value input by PC, and a spectrum of each frequency is provided. Using those spectrum, we detect the coordinate of the light by calculation.

Defines the coordinates of each LED in advance

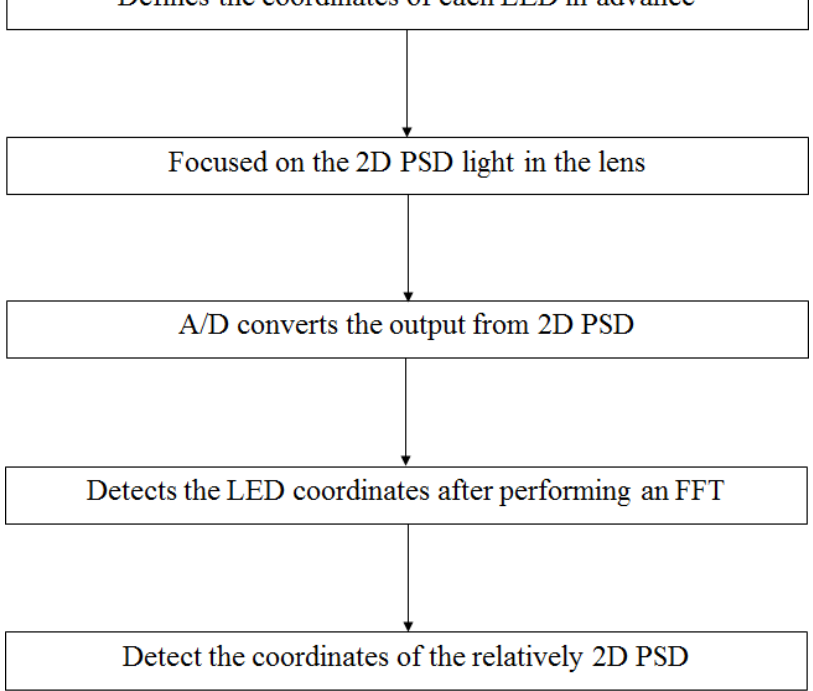

Fig.4. The series of flow 
We measured the position coordinates of the moving objects which carried PSD under various condition. We conduct the experiment under the following conditions.

- Frequency interval

- The sampling frequency $: 25[\mathrm{kHz} / \mathrm{ch}]$

- Beam angle of LED $: 140\left[^{\circ}\right]$

- Distance from the 2D PSD to the lens : $4.5[\mathrm{~cm}]$

\subsection{The measurement using four LED}

We performed the position detection of the moving objects using four LED as a preliminary experiment. The light that PSD receives does not change because the LED is only four. We show a figure of image of the experiment in the Fig.5. Frequency of each LED and placement are just what to show it in Fig.5.

We detected $Y$ coordinate of the PSD side while fixing $X$ coordinate of the PSD side, and moving $Y$ coordinate every $20[\mathrm{~mm}]$ from a point of $-100[\mathrm{~mm}]$ to a point of $100[\mathrm{~mm}] . X$ coordinate was set $-60[\mathrm{~mm}],-30[\mathrm{~mm}], 0[\mathrm{~mm}], 30[\mathrm{~mm}]$, $60[\mathrm{~mm}]$. We show the Y coordinate measurement result when the $X$ coordinate was $-60[\mathrm{~mm}], 0[\mathrm{~mm}], 60[\mathrm{~mm}]$ in Fig. 6 (a), (b), (c).

\section{Experiments} The distance from LED to optical lens assumed it $2.0[\mathrm{~m}]$.

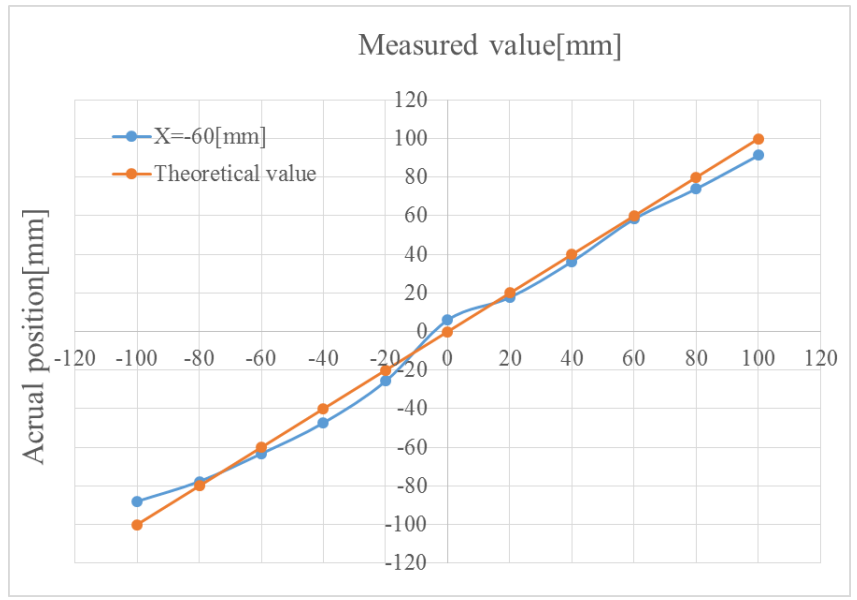

(a) $X=-60[\mathrm{~mm}]$

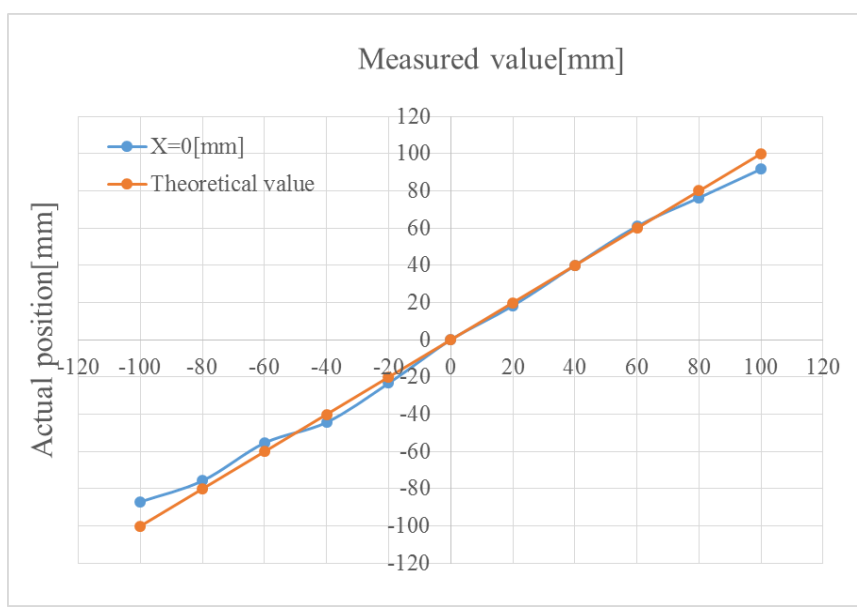

(b) $X=0[\mathrm{~mm}]$

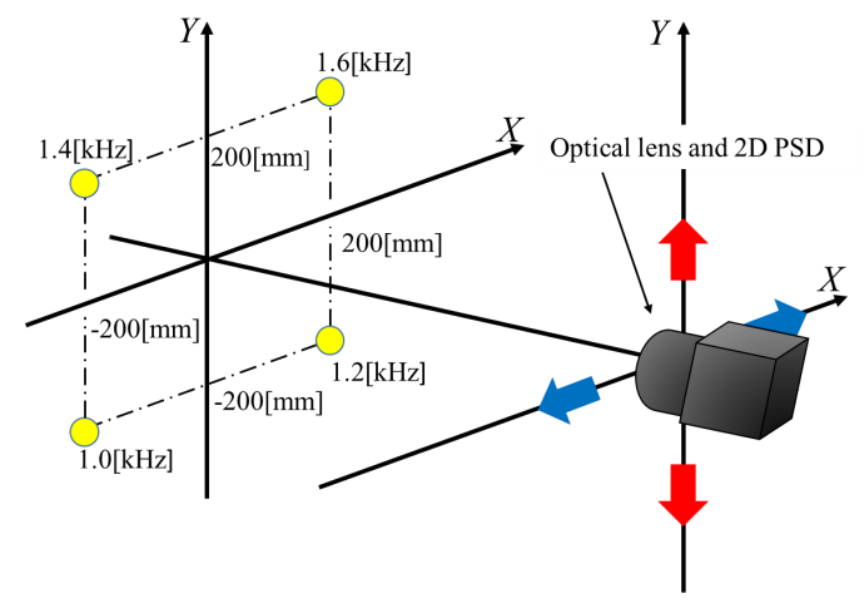

Fig.5. Experiments image using four LEDs

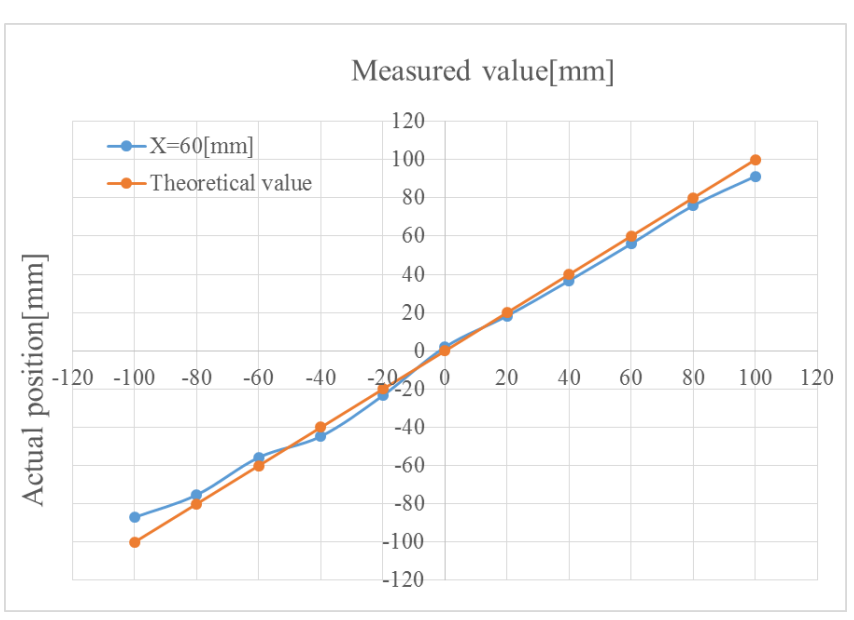

(c) $X=60[\mathrm{~mm}]$

Fig.6 Measurement result of experiments using 4 LEDs 
The result of a measurement and the theoretical value were about the same, and it was revealed that position detection was possible by this method.

\subsection{The measurement using six LEDs}

We performed the position detection of the moving objects using six LEDs. Because this measurement was aimed for the position detection of the moving objects, We performed position detection in the situation that change of the light that PSD receives in the case of the movement and choice of the light to use for operation produced. We show a figure of image of the experiment in the Fig.7. Frequency of each LED and placement are just what to show it in Fig.7. We detected $Y$ coordinate of the PSD side while fixing $X$ coordinate of the PSD side, and moving $Y$ coordinate every $50[\mathrm{~mm}]$ from a point of $-200[\mathrm{~mm}]$ to a point of $200[\mathrm{~mm}] . X$ coordinate was set $-50[\mathrm{~mm}], 0[\mathrm{~mm}], 50[\mathrm{~mm}]$ and the distance from LED to optical lens assumed it 2.0[m], $2.2[\mathrm{~m}]$. We show the $\mathrm{Y}$ coordinate measurement result when the $X$ coordinate was $-50[\mathrm{~mm}], 0[\mathrm{~mm}], 50[\mathrm{~mm}]$ and the distance from LED to optical lens was 2.0[m] in Fig. 8 (a), (b), (c). We show the Y coordinate measurement result when the $X$ coordinate was $-50[\mathrm{~mm}], 0[\mathrm{~mm}], 50[\mathrm{~mm}]$ and the distance from LED to optical lens was 2.2[m] in Fig. 9 (a), (b), (c).

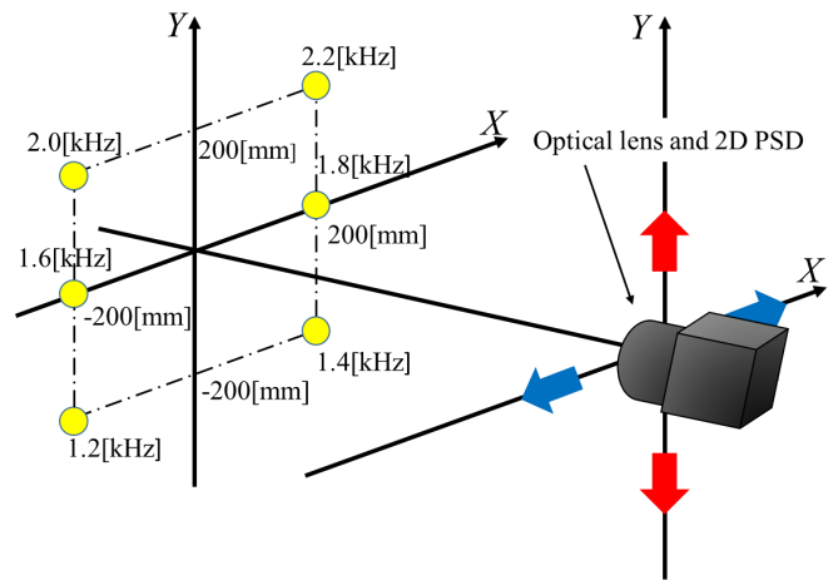

Fig. 7 Experiment image using six LEDs

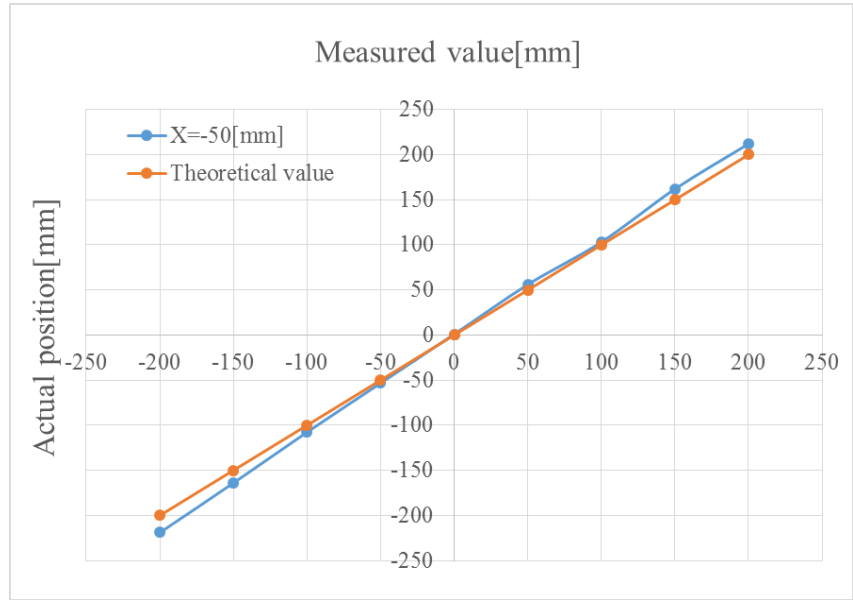

(a) $X=-50[\mathrm{~mm}]$

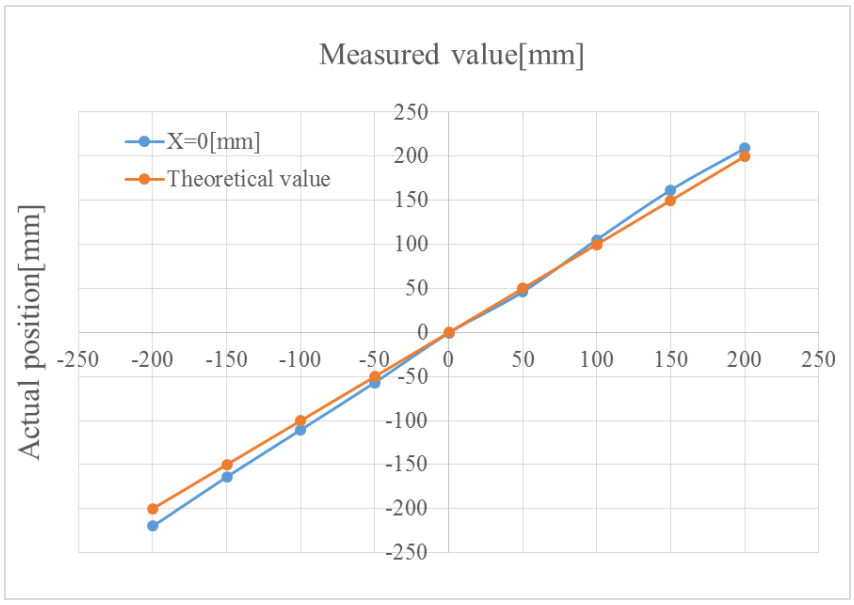

(b) $X=0[\mathrm{~mm}]$

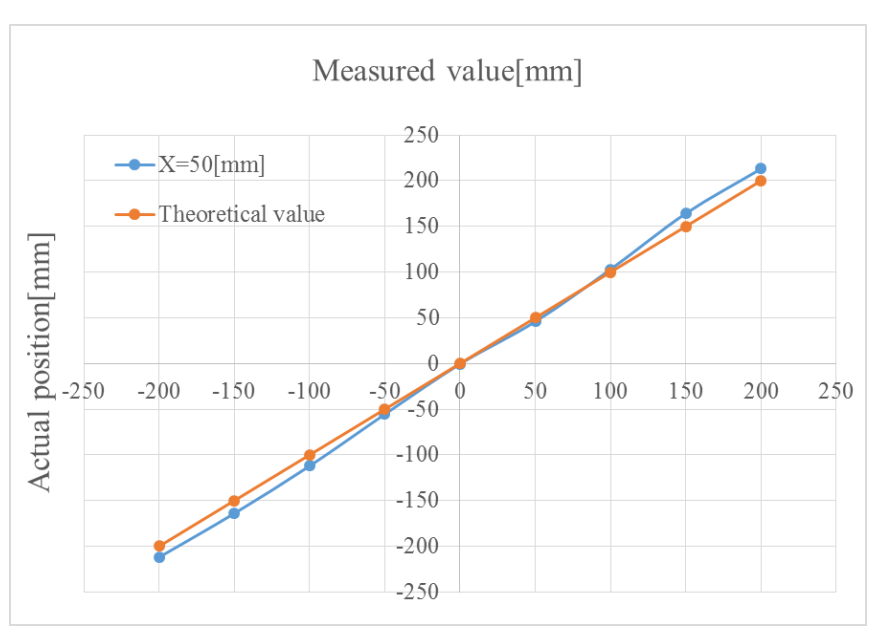

(c) $X=50[\mathrm{~mm}]$

Fig. 8. Measurement result of experiments using 6 LEDs (Distance from the LEDs to the lens : $2.0[\mathrm{~m}]$ ) 


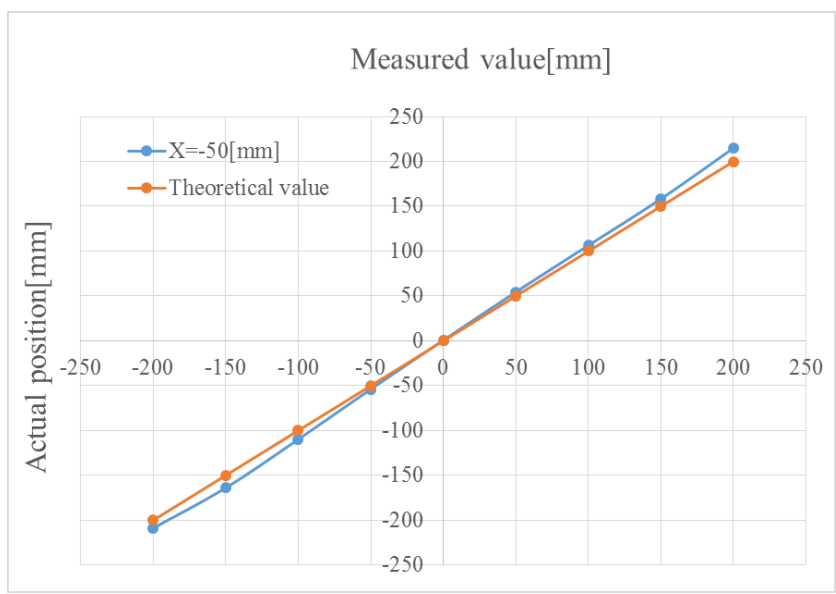

(a) $X=-50[\mathrm{~mm}]$

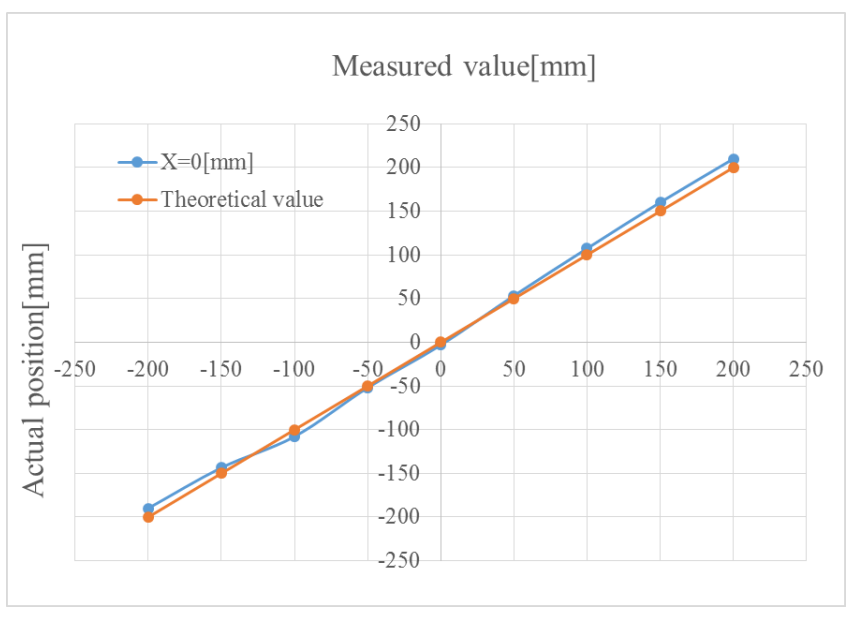

(b) $X=0[\mathrm{~mm}]$

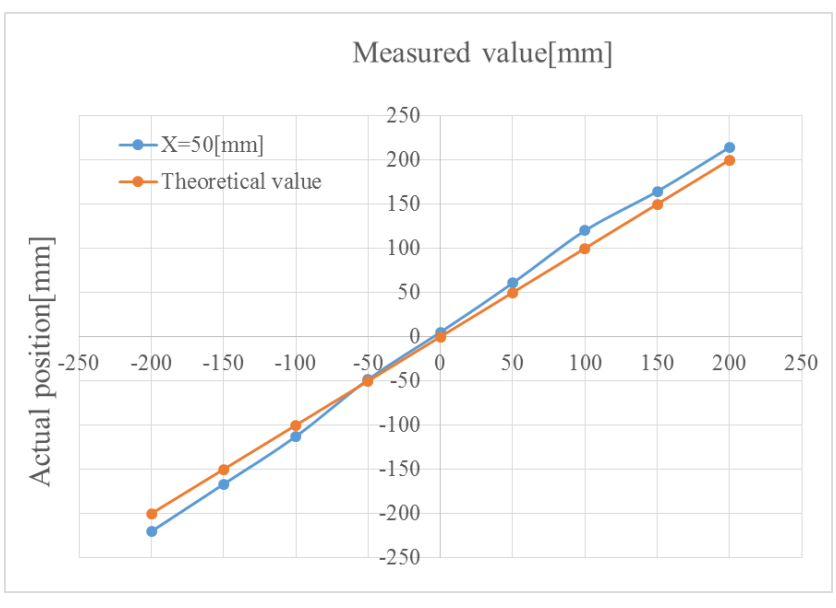

(c) $X=50[\mathrm{~mm}]$

Fig. 9. Measurement result of experiments using 6 LEDs (Distance from the LEDs to the lens : 2.2[m])
The result of a measurement and the theoretical value were about the same, and it was revealed that position detection of the moving objects was possible by this method. There were not many changes even if we narrowed distance of the LEDs. There is the part which an error comes to have a big so that the distance from the origin goes far, it is thought that an error of distance from distance and the optical lens from LED to an optical lens to PSD influences this. It is thought that the reason why result of a measurement is different is that strength by the decrement of the light when it was subjected to light by PSD is different from the angle that can condense light according to distance.

\section{Conclusion}

In this study, we proposed the new method of position detection of the moving objects using a 2D PSD and the ceiling LED lighting. This method could do accurate position detection of the moving objects and could use the ceiling LED lighting that we always used and showed that it was position detection indoor, not to need the new facilities such as landmarks. Position detection using plural LED is enabled at the same time by using FFT and frequency modulation. Because It is not influenced by neighboring scenes and some indoor layout changes, this method is available under various situation. Distinction of ambient light and the light of the LED is possible by performing frequency modulation. It is future work that the expansion of the measurement range or the detection of the degree of leaning.

\section{References}

(1) Fabien Launay, Akihisa Ohya and Shin'ichi Yuta: "A Corridors Lights based Navigation System including Path Definition using a Topologically Corrected Map for Indoor Mobile Robots", Proceedings 2002 IEEE International Conference on Robotics and Automation, pp.3918-3923 (2002)

(2) Masako Kumano, Akihisa Ohya and Shin'ichi Yuta: "Obstacle Avoidance of Autonomous Mobile Robot using Stereo Vision Sensor", Proceedings of the 2nd International Symposium on Robotics and Automation, pp.497-502 (2000)

(3) Shiyuan Yang, Kiyotada Matsura, Shun Inoue, Fenghui Yao, "Method of Concurrent Detection for Multiple 
Light Sources Position by Using the PSD",

International Conference on Industrial Application

Engineering 2013, pp.273-276 (2013) 